\title{
TITLE:
}

\section{A New Species of Hansenocaris (Crustacea : Facetotecta) from Tanabe Bay, Japan}

\author{
$\operatorname{AUTHOR}(\mathrm{S}):$
}

Ito, Tatsunori

\section{CITATION:}

Ito, Tatsunori. A New Species of Hansenocaris (Crustacea: Facetotecta) from Tanabe Bay, Japan. PUBLICATIONS OF THE SETO MARINE BIOLOGICAL LABORATORY 1989, 34(1-3): 5572

\section{ISSUE DATE:}

1989-08-31

URL:

http://hdl.handle.net/2433/176158

RIGHT: 


\title{
A New Species of Hansenocaris (Crustacea: Facetotecta) from Tanabe Bay, Japan
}

\author{
By \\ Tatsunori Itô \\ Seto Marine Biological Laboratory, Kyoto University, \\ Shirahama, Wakayama 649-22, Japan
}

With Text-figures $1-7$

\begin{abstract}
A new species of "cypris y" (Crustacea: Facetotecta) is described from Tanabe Bay on the Pacific coast of Japan under the name Hansenocaris furcifera. The nauplius of this new species is "nauplius y type IX" sensu Itô. A possible penis is recognized in the Facetotecta for the first time, represented by an apically bifurcate process on the ventum of the first abdominal somite. External features of various body parts are described in detail based upon observations with a scanning electron microscope. New terms are proposed to describe certain structures.
\end{abstract}

The four named species of the Facetotecta Grygier (Crustacea: Maxillopoda) that are known from the so-called "cypris y" stage are currently accommodated in the single genus Hansenocaris Itô, which was originally based upon Japanese cypris y larvae (Itô, 1985, 1986). In the present paper I describe another new species of Hansenocaris from Tanabe Bay on the Pacific coast of Honshu, the main island of Japan. In order to refine the ordinary description based upon light microscopy, a detailed study of its morphology by scanning electron microscopy has been carried out. The description presented here is based upon only the cypris y stage, but it has already been determined by me that its nauplius belongs to type IX sensu Itô (1987b). Its naupliar development will be reported in a forthcoming paper.

The cyprid specimens used for this study were raised from nauplius (metanauplius) larvae by individual culture in the laboratory (method of Itô \& Takenaka, 1988). The nauplius larvae were collected from plankton samples taken in Tanabe Bay off the Seto Marine Biological Laboratory $\left(33^{\circ}\right.$ $42>135^{\circ} 21^{\prime} \mathrm{E}$ ). Naupliar exuviae left in culture vessels after molting were used for species identification. Where data are given in the following text as a range of dates, the first and last days are the ones when a nauplius was collected for laboratory culture and the one when its metamorphosed cyprid was fixed, respectively. All specimens were fixed in 5\% Formalin-sea water and kept in glycerin. Dissected appendages were mounted onto slides with gum-chloral medium, while isolated abdomens were mounted in anhydrous glycerin. The type series is deposited in the Seto Marine Biological Laboratory.

Three specimens for SEM study were dehydrated through a graded series of ethanol, transferred into isoamyl acetate, and dessicated in a critical-point drier using carbon dioxide. Dried specimens were sputter-coated with gold and observed in an SEM (JEOL, JSM T-220) at an accelerating voltage of $10 \mathrm{kv}$. These specimens were not included in the type series.

The terminology used herein is adopted mainly from Itô (1985). In this new species, the last abdominal somite is nonagonal in cross section, composed of four pairs of symmetrical planar surfaces

Publ. Seto Mar. Biol. Lab., 34(1/3), 55-72, $1989 . \quad$ (Article 5) 
and one unpaired surface which forms the ventral face. The nine planar surfaces and the structures on them (e.g., plates) will be specified as dorsal, dorsolateral, lateral, ventrolateral, and ventral (see Fig. 1F).

\section{Hansenocaris furcifera sp. nov.}

Type series. Holotype (dissected) : cypris y metamorphosed in the laboratory from a nauplius collected from a plankton sample $(23 \sim 26-\mathrm{V}-1986)$. Paratypes: two individuals of cypris y, both metamorphosed in the laboratory (paratype-1, dissected, 23 26-V-1986; paratype-2, intact, 21 25VII-1986). Type locality: Tanabe Bay, Wakayama Pref., Japan.

Description of the holotype: Body (Fig. 1A, B; see Fig. 4A) about $420 \mu \mathrm{m}$ long, consisting of a cephalon, six thoracic somites with paired legs, four abdominal somites, and furcal rami; colorless and semitransparent except for eyes and inclusion within last abdominal somite. Carapace $350 \mu \mathrm{m}$ long, $150 \mu \mathrm{m}$ wide, extending over third thoracic somite laterally, decorated laterally with mostly longitudinal lines or low ridges on its smooth surface (Fig. 2B; see Fig. 4A, B); ventral rims inrolled (Fig. 2A; see Figs 2E and 4B, D). A prominent pair of compound eyes, each eye located lateral to labrum, with red pigment. Nauplius eye located anterior to labrum, red. Paraocular process (Fig. 2A; see Figs 2E and 4B, D) bifurcate, both branches about $30 \mu \mathrm{m}$ long. Naupliar second antennae and mandibles remaining as small, crumpled protuberances (Fig. 2A; see Figs 2E, 5B). Postocular filamentary tuft not detected. Labrum (Fig. 2A, C) armed with a single apical claw and two pairs of subapical, posterior claws; posterior edge formed into an almost conical protuberance terminating in a pore; proximal portion composed of soft cuticle, bearing a pair of widely spaced pores posteriorly.

Tergites of first four thoracic somites lacking pleural extentions, while tergites of last two thoracic somites bilaterally produced into pleurae (Fig. 1B). First thoracic somite markedly shortened toward its dorsum, not clearly definable anteriorly except for its ventum. Other ornamentation of thoracic somites will be dealt with in detail later.

First three abdominal somites almost the same in length as well as in width; each somite covered with a well-developed pleurotergite continuous with sternite; each pleurotergite fringed posteriorly with a hyaline membrane, which appears to be devided in places by continuations of the arched ridges; pleural area of second and third abdominal somites markedly extending toward rear. First abdominal somite armed ventrally with a single, prominent process identified as a penis (Fig. 1D, E; cf. Figs 5D and 6). Penis sclerotized, with no articulation separating it from sternite, bifurcate apically to form two knife-edges; pair of lateral grooves extending along its anterior (ventral) edge, which terminates in a fine point. Midposterior portion of sternite of second abdominal somite slightly extending toward rear, apically bifid.

Last abdominal somite longer than three preceding ones combined, well sclerotized, somewhat depressed dorsoventrally, containing sausage-shaped mass of brownish pigment internally (see Fig. 1B); surface without mesh-like texture, covered with more or less rectangular plates delineated by chitinous ridges; dorsal plates 


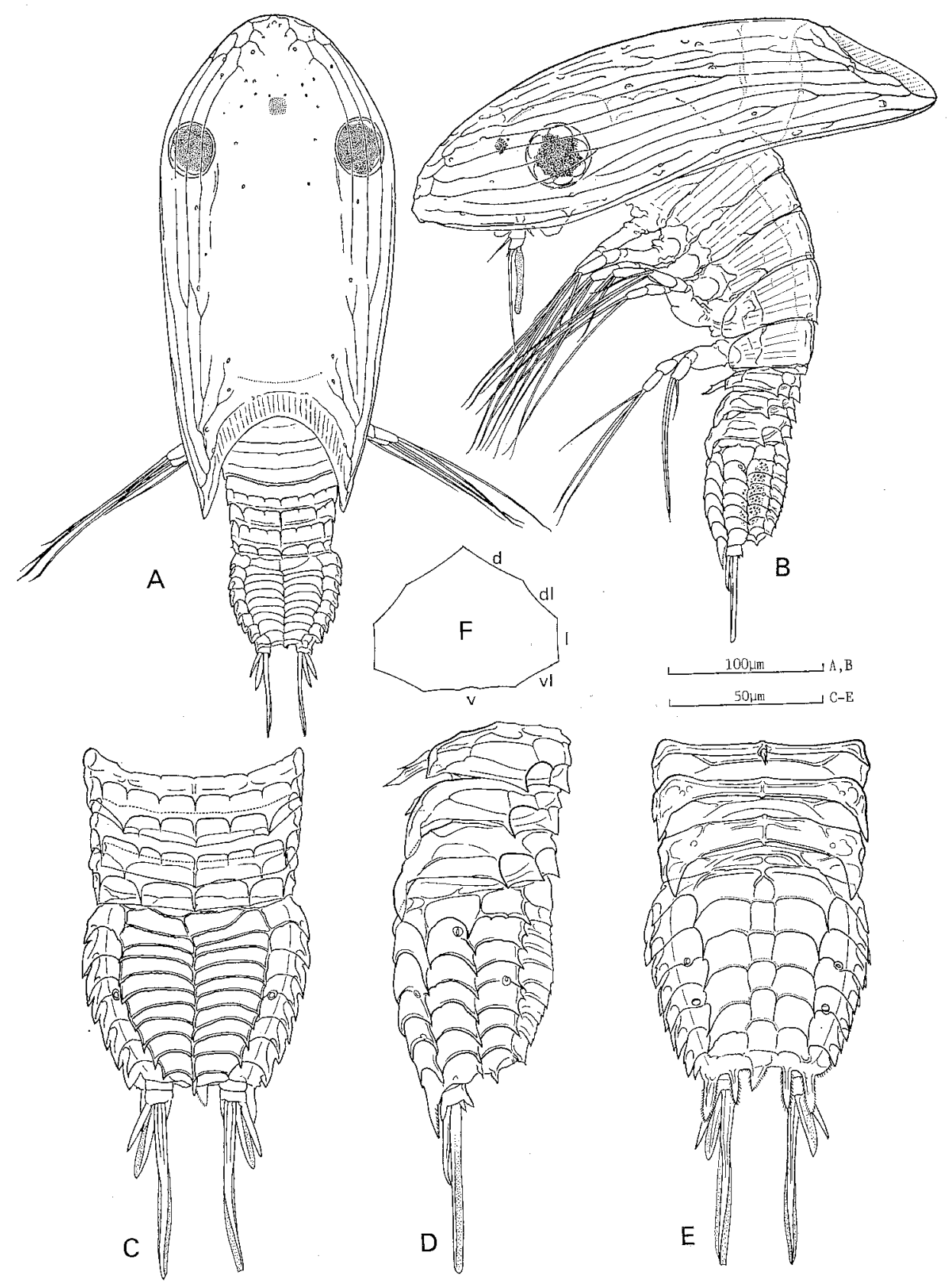

Fig. 1. Hansenocaris furcifera sp. nov. A-E, holotype. A. Habitus, dorsal; B. Habitus, lateral; C, D, and E. Dorsal, lateral and ventral views of abdomen, respectively. F, schematic representation of cross section of last abdominal somite: $\mathrm{d}$, dorsal; $\mathrm{dl}$, dorsolateral; 1 , lateral; vl, ventrolateral; v, ventral. 
separated into two rows by median carinate ridge, 10 plates on left, 11 plates on right, most plates rectangular and laterally extended (Fig. 1G; see Fig. 5C); seven plates recognized on each dorsolateral surface, accompanied by a prominent "pore" (described in detail later) on the ridge between the fourth and fifth ones; at least six plates occurring on each lateral surface, accompanied by a prominent "pore" at anterodorsal corner of the second one (Fig. 1D); at least six plates occurring on each

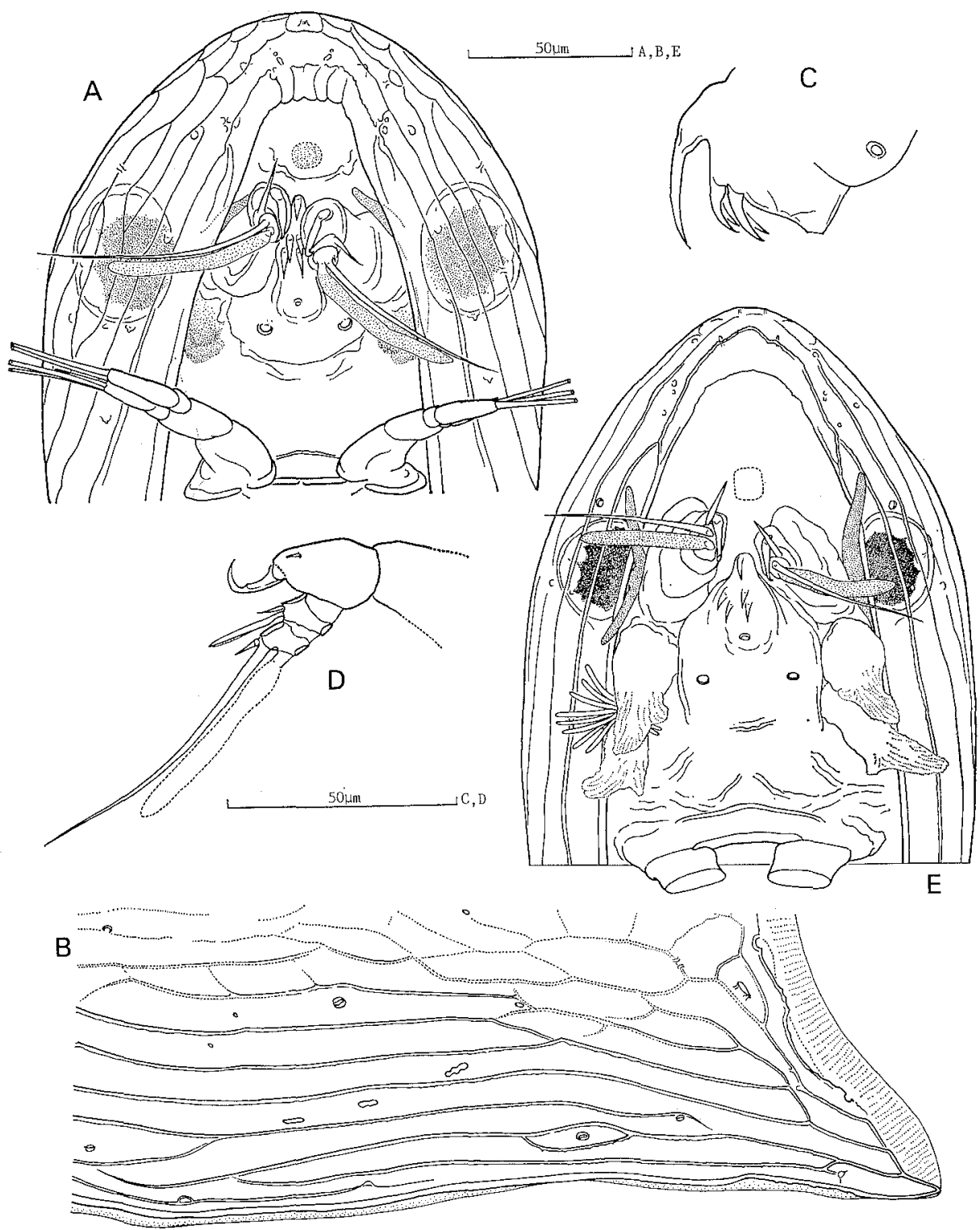

Fig. 2. Hansenocaris furcifera sp. nov. A-D, holotype. A. Cephalon and first leg, ventral (foreshortened due to angle of view); B. A ventral portion of carapace; C. Labrum; D. First antenna (apical aesthetasc was broken during dissection). E, paratype-2. Cephalon and first leg, ventral. 

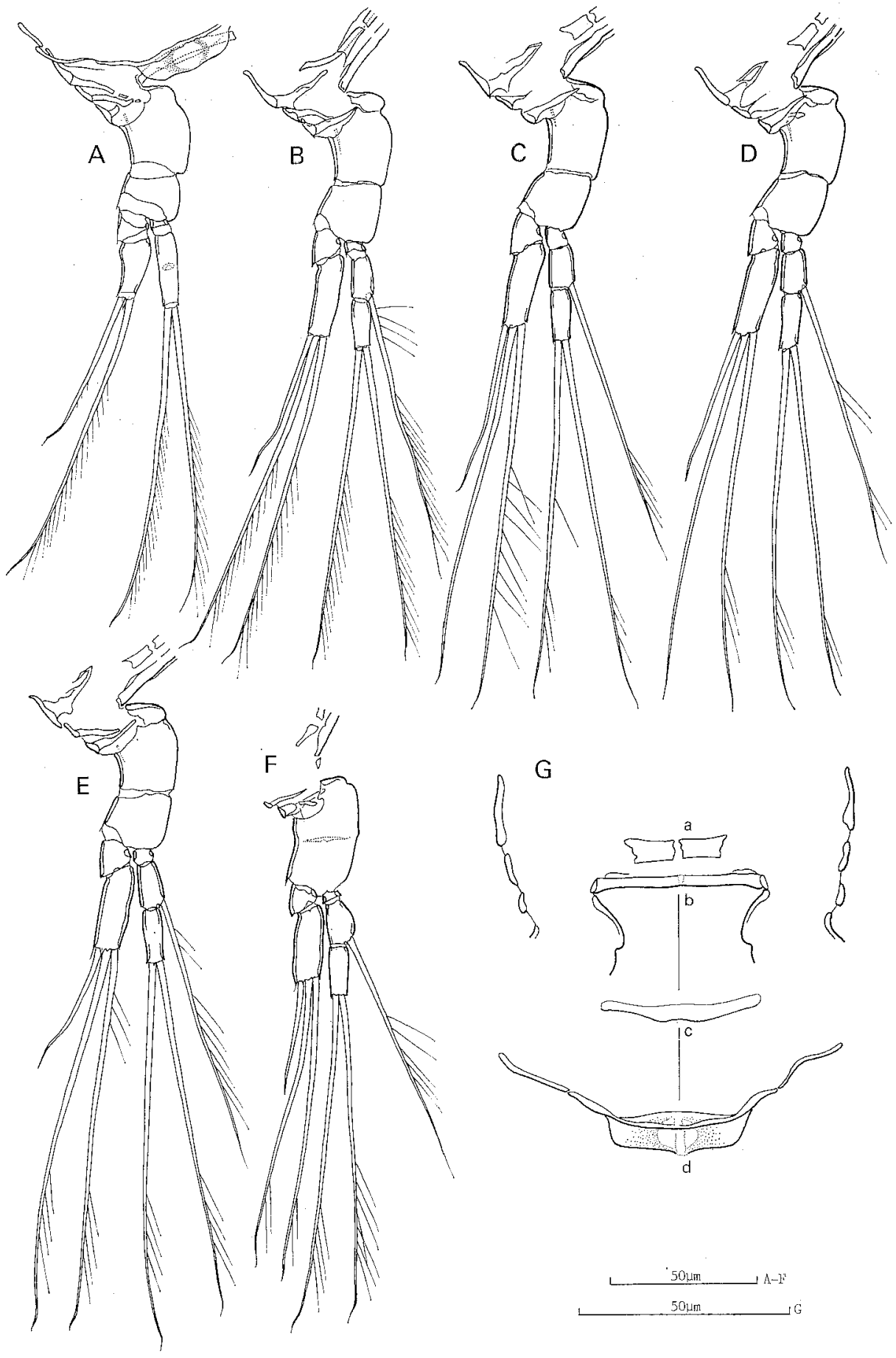

Fig. 3. Hansenocaris furcifera sp. nov. Holotype. A. Leg 1, posterior; B. Leg 2, anterior; C. Leg 3, anterior; D. Leg 4, anterior; E. Leg 5, anterior; F. Leg 6, posterior; G. Intercoxal plate and associate structures of leg 3, viewed in a phase-contrast microscope by changing its forcus from anterior plane (top) to posterior plane (bottom). Labels a-d are used in the text as reference marks. 
ventrolateral surface, accompanied by two "pores," one located close to the posterior edge of the third plate, the other close to the posterior edge of the fifth plate (Fig. IE); ventral surface delimited by low ridges into three rows of plates, each row consisting of six plates, median row narrower than others; five lanceolate processes arising from posterior end of ventral face, all bilaterally serrate, the median one shortest. Furcal rami two-annulated (Fig. 1C); each ramus with short, spiniform projections posteriorly, armed with three terminal setae, all fringed with membranous structures, the innermost longest and as long as last abdominal somite.

First a n t en n a (Fig. 2A, D; see Fig. 4B-D) four-segmented; first segment with no armature, cuticle of its proximal half appearing soft; second segment extending anterodistally, armed with a strong, arched claw on its tip and a short seta on subapical outer side; third and fourth segments short, subequal in length; third segment armed with two juxtaposed setae on its anterior end; fourth segment apically armed with one setule, one long seta and one well-developed aesthetasc (that in Fig. 2D was broken during dissection).

L e g 1 (Fig. 3A). Intercoxal plate and associated structures are described in detail later. Coxa clearly demarcated from basis, with three outer sclerites proximally, a spinule-like, internal structure close to its outer edge basally. Exopod two-segmented; first segment unarmed, shorter than second segment; second segment armed with two apical setae, outer one shorter; setal tips appearing rigid, somewhat sinuate, and probably finely serrate (not illustrated). Endopod twosegmented; first segment very short, with no seta; second segment ornamented with a small depression on its mid-posterior surface (not scar of detached seta), armed with two apical setae, outer one appearing to have a rigid tip.

L e g 2 (Fig. 3B), 1 e g 3 (Fig. 3C, G), 1 eg 4 (Fig. 3D), l e g 5 (Fig. 3E). Coxa, basis, and segmentation of exopod as in leg 1 . Second exopod segment armed with one additional, apical seta (three in total), which appears to have a rigid tip. Endopod three-segmented, first segment very short, other two subequal in length; first segment with no seta; second segment armed with one inner seta, which does not appear to have a rigid tip; third segment armed with two apical setae as those on second endopod segment of leg 1 .

L e g 6 (Fig. 3F). Coxa with at least two outer sclerites proximally, demarcated from basis by a very faint suture, shorter and wider than counterparts in preceding legs. Exopod two-segmented, armed with three apical setae on its second segment. Endopod three-segmented and armed with one and two setae on second and third segments, respectively, as in preceding four legs; inner edge of second segment apparently swollen.

Paratypes and detailed observations using SEM. Body of paratype-1 $450 \mu \mathrm{m}$ long, paratype-2 $470 \mu \mathrm{m}$ long. Since the paratype-2 was fixed immediately after the molt into the cypris $y$ stage, the naupliar second antennae and mandibles remain as prominent processes (Fig. 2E). In this specimen, at least nine filaments of the right postocular filamentary tuft are clearly seen between the vestiges of the right 

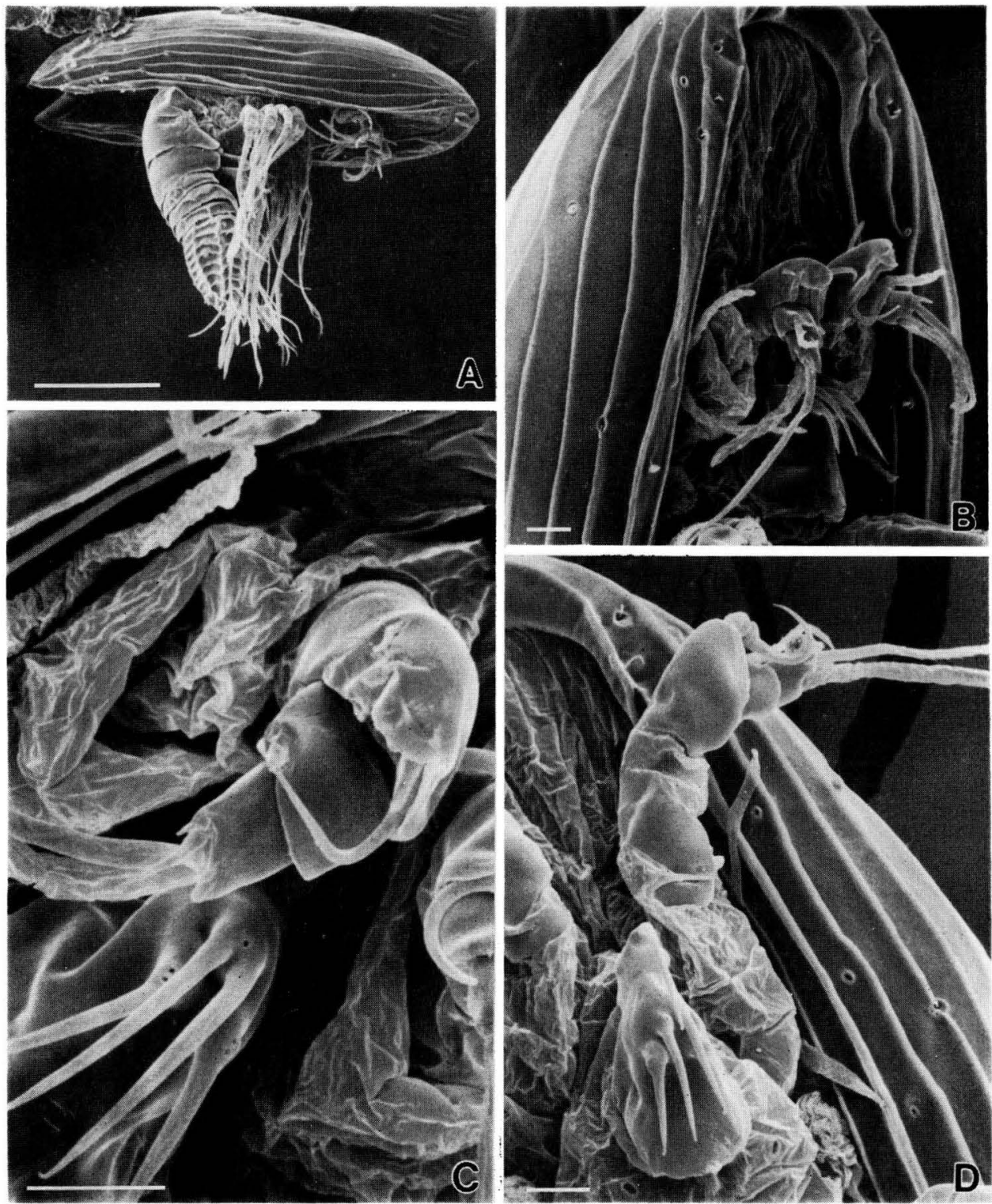

Fig. 4. Hansenocaris furcifera sp. nov. SEM photomicrographs (A-C were based upon a single specimen, D was based upon a separate specimen). A. Habitus, ventrolateral; B. Ventrolateral view of cephalon; C. Closer looks of first antenna (a seta of subapical segment was broken off) and labrum with pores; D. Ventral view of cephalon, showing the general shape of first antenna. Scale: $10 \mu \mathrm{m}$.

second antenna and mandible, while no filaments are detected on the other side. The vestigial, naupliar second antennae and mandibles as seen with SEM have crumpled surfaces (Fig. 5B). No part of the postocular filamentary tufts is recognized in the specimens observed with SEM, while paraocular processes are clearly 

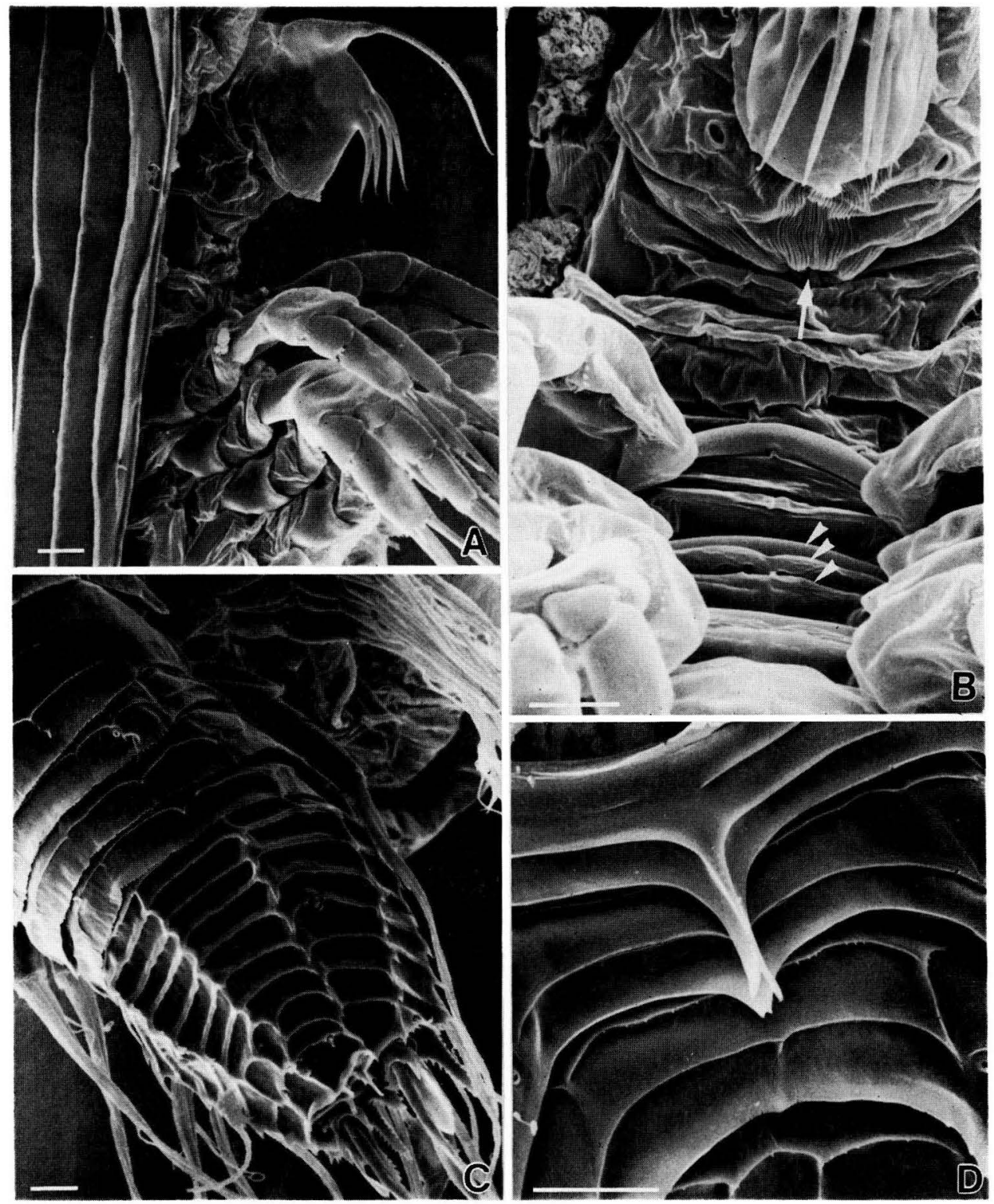

Fig. 5. Hansenocaris furcifera sp. nov. SEM photomicrographs. A. Lateral views of labrum with long apical claw and legs (coxicules of each legs are seen); B. Ventral view of cephalon and thorax (vestiges of naupliar second antenna and mandible are present beside labrum, and intercoxal plate and associate structures are seen between paired legs of each thoracic somite) (arrow indicating mouth; arrowheads indicating intercoxal plate, intercoxal ridge, and posterior ridge of sternite of second thoracic somite); C. Dorsolateral view of abdomen; D. Ventral view of penis (apically bifid, medial extention of sternite of second abdominal somite is seen beneath apical portion of penis). Scale: $10 \mu \mathrm{m}$. 
seen (Fig. 4).

The anterior outline of the illustrated carapace of paratype- 2 is seemingly different from that of the holotype (cf. Fig. 2A and 2E). Actually, this is due to a difference in the orientation of the specimens under the microscope. Fixed specimens of this species, including the holotype, usually exhibit a bowed posture, as shown in Fig. 1B and Fig. 4A, which makes it difficult to place their head on a slide glass horizontally.

It is seen with SEM that the carapace is equipped with sensory hairs as well as short, tubular processes with pores. Some of the tubular processes are almost regularly arranged along the ventral rim of carapace (Fig. 4B, D).

The thoracic legs of the dissected paratype agree with their counterparts in the holotype not only in the segmentation, but also in the setal and spinal armature. The segmentation and spines of the intact paratype are also the same as in the holotype; however, not all of its setae could be precisely counted due to overlapping limb elements.

A penis like that of the holotype was observed in both paratypes as well as in other specimens observed with SEM (Figs 5D, 6). No particular difference was found among their penes. When a penis is viewed laterally in the light microscope, its anterior edge appears to be clearly separated from the posterior part by a deep incision (see Fig. 1D). However, in SEM observation, the incision is not so deep. Actually, a groove is present on both sides of the penis along its thick, anterior edge and these grooves form a longitudinal thin zone that have appeared as the deep incision of the penis in light microscopy.

In paratype- 1 the two rows of dorsal plates on the last abdominal somite consist of 10 plates each. However, the median ridge that should separate the anteriormost pair of plates is so inconspicuous that only nine completely bounded plates are counted in each row. In paratype-2, the rows of dorsal plates consist of 10 plates each. In a specimen examined with SEM (Fig. 5C), 10 plates were regularly arranged in the

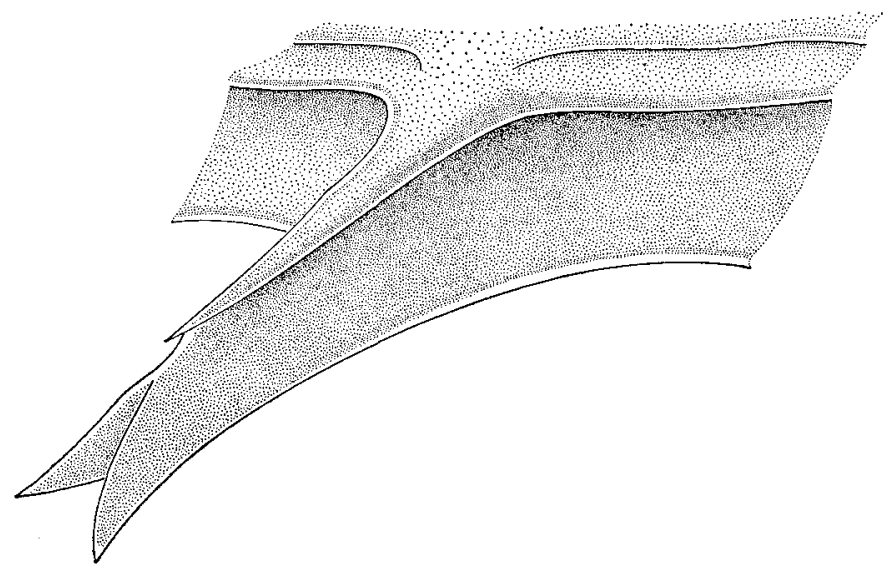

Fig. 6. Hansenocaris furcifera sp. nov. Schematic illustration of penis. 
right row, while the posterior plates of the left row, also consisting of 10 plates, appear irregular; the area corresponding to the combined eighth and ninth plates of the right row is split by a longitudinal ridge rather than a transverse one. Ten dorsal plates per row seem to be standard in this new species.

The last abdominal somite bears at least two pairs of hairs that arise from pits, one pair located on the ridges between the ninth and tenth plates, the other pair located near the posterior edges of the lateral faces (Fig. 5G). These hairs are not always apparent in light microscopical observations since they are hidden against a complex background. At least two pairs of thick hairs or hairlike processes are recognized with SEM to arise from the rear end of the last abdominal somite, a little above the level of the furcal rami (Fig. 5C).

Prominent "pores" like those recognized on the last abdominal somite of the holotype are also found in the paratypes. According to SEM examinations, each "pore" is actually a hemispherical depression encircled by a ridge, within which a tubular protuberance occurs (Fig. 5G).

The most apical claw of the labrum may be somewhat variable in shape. In a specimen observed with SEM, it appears to be markedly slimmer and longer than in the holotype (Fig. 5A). Very small pores (less than $0.8 \mu \mathrm{m}$ in diameter), which were not detected in light microscopical observation, occur on the labrum; one is located between the paired anterior (ventral) claws, and the others are located on the proximal portion of each paired, posterior (dorsal) claw (Fig. 4C).

The proximal portion of rear surface of the labrum consists of soft, finely and densely wrinkled cuticle (Fig. 5B). There appears to be a deep depression just above or within this wrinkled area, which must be the mouth.

The arched claw on the secnod segment of the first antenna is apparently movable because its position relative to the segment is different among specimens or even in a single specimen (cf. Fig. 4B-D). The apicomedial portion of the second segment extends along the third segment to form a deep recess, into which the claw is retractable (Fig. 4G).

The tergites of the first four thoracic somites have no pleural extensions. Each of these tergites has a clear incision of its posterolateral edge (Fig. 7A). A narrow, rectangular, sclerotized plate sits in the area formed by this incision. I call this plate the "pleural slat." It is connected with the incised edge of the corresponding tergite by a soft articulation membrane. The ventral portion of each pleural slat extends into the narrow space between the leg of its somite and that of the succeeding somite, close to the rear side of the former. The pleural slat seems to be usually situated on the trunk so that its free, external surface faces laterally or somewhat lateroventrally. Where the thorax is bent, however, it appears to become rotated along its long axis so its free surface faces anteriorly. The pleural slat of the fourth thoracic somite shown in Fig. 7A is so rotated. It appears as though it had been pushed to the rear. Its free surface faces front, and its ventral portion appears to be appressed to the rear side of the proximal portion of the coxa of leg 4 . There is little doubt that the pleural slat is not directly jointed to any sclerotized elements of the trunk or limbs 
surrounding it, even though it is connected to the latter with articulation membranes. In light microscopical observation, the pleural slats are occasionally recognized as sclerotized bars or chords in association with dissected legs (Fig. 3A).

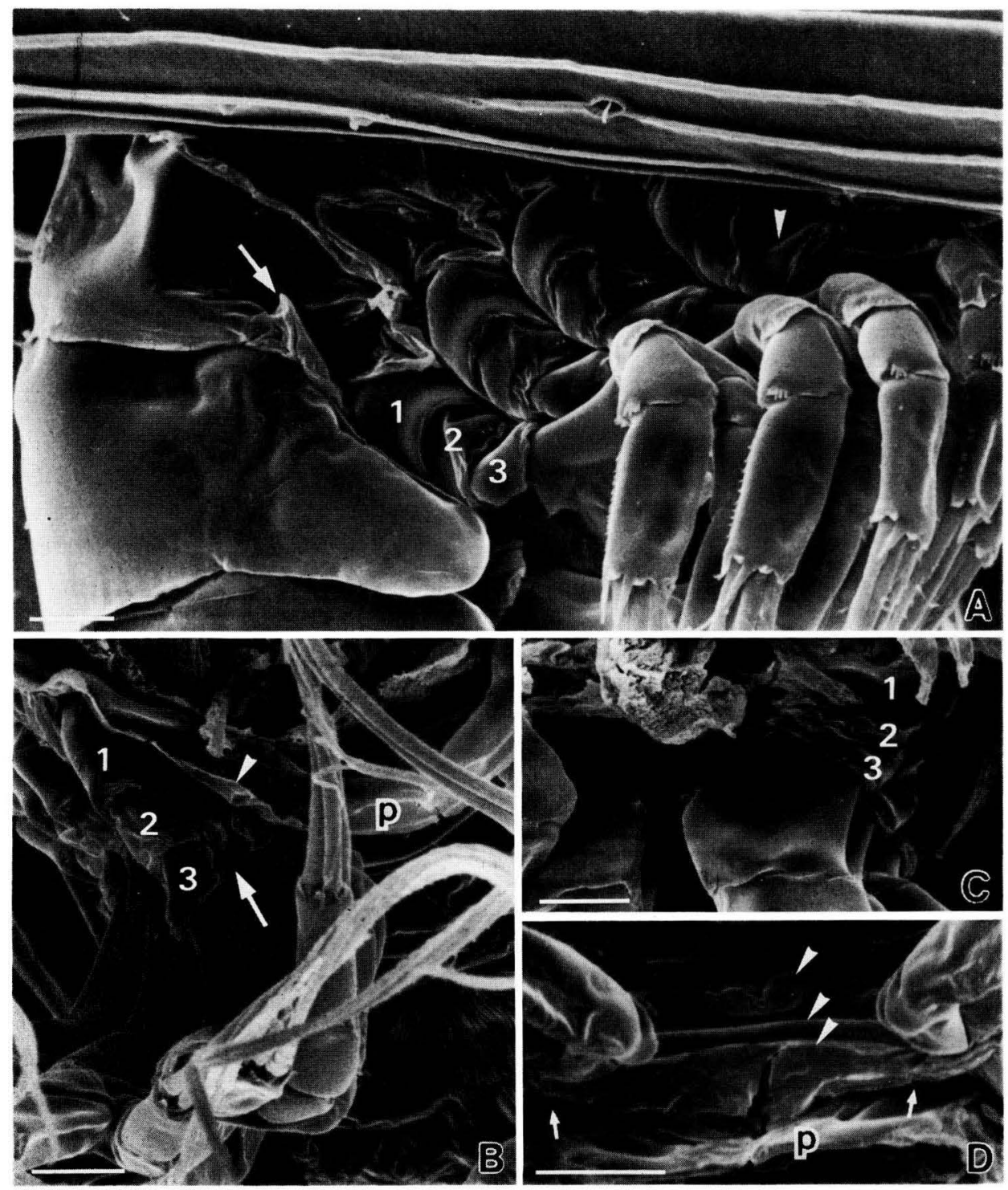

Fig. 7. Hansenocaris furcifera sp. nov. SEM photomicrographs. A. Lateral view of thorax (arrow indicating pleural slat of fourth thoracic somite; arrowhead indicating pit of third coxicule of leg 1); B. Posterior view of fourth leg (arrow indicating pivot hole; arrowhead indicating rolled edge of posterior ridge of sternite); C. Anterior view of proximal portion of leg 2; D. Ventral view of first thoracic somite (arrows indicating paired depressions of sternite; arrowheads indicating anterior lobe (uppermost), intercoxal plate, and intercoxal ridge of sternite. 1-3, first, second and third coxicules; p, posterior ridge of sternite. Scale: $10 \mu \mathrm{m}$. 
In lateral view, each coxa of at least first five pairs of legs appears to be proximally equipped with three seemingly annular parts (Figs 5A, 7A), which I would like to call, from basal to distal, the "first, second, and third coxicules." In anterior view, the coxa of each leg appears to have a flexible basal area that consists of the membranous portions of the connected coxicules (Fig. 7C). This area looks like a bellows and occupies most of the width of the anterior base of coxa. More distal areas of the anterior side of coxa appear to be almost evenly sclerotized except for a flaw that arises from the inner distal limit of the above-mentioned flexible area and extends to the distal limit of the coxal boss (see Figs 3B-D, 7C). In contrast, in a leg viewed from the rear, the sclerotized, major part of the coxa appears to have a precisely delineated, $\mathrm{v}$-shaped incision at its outer base. There is a prominent, deep hole within the area bordered by the edges of this incision (Fig. 7B). I call it the "pivot hole." The posterior portions of the three coxicules taper inwards, and meet to form the wall of this pivot hole. There is no such hole on the anterior face of coxa.

In each of the first four thoracic somites, the first coxicule is clearly delimited from the tergite of the corresponding somite by an intervening, wide articulation membrane of soft cuticule. In the last two thoracic somites, the first coxicule is connected to an internal protuberance of the pleurotergite by a relatively narrow articulation membrane. This internal protuberance marks the proximal border of the pleural area of each pleurotergite. Most of the first coxicule is sclerotized. Its lateral edge extends dorsally to form a short process (Fig. 7B), and its posterior portion is interrupted by a slit just before the pivot hole. Thus, there is a small, triangular sclerite that is connected with the rest of the first coxicule by a narrow membrane.

The second coxicule appears as if it were inserted into the first coxicule. Its anterior to distolateral portion is not sclerotized, but is made of flexible membrane. No articulation membrane between the first two coxicules is obvious posteriorly; rather, the distal edge of the first coxicule and the basal edge of the second are closely set against each other, forming a narrow groove, which continues as part of the pivot hole's wall. A few spinules occur near the distal edge of the anterolateral side of the second coxicule (Fig. 7A).

The third coxicule extends medially beyond the midwidth of the anterior side of coxa. There is a shallow pit on the lateral side (Fig. 7A). The posterior portion of this coxicule is small, and the inner portion, which is probably not sclerotized, curves into the pivot hole as part of the hole's wall.

When a successfully dissected pair of legs is examined with a microscope, the legs appear to be connected by an intercoxal plate (for terminology see Itô, 1986) together with other, more or less plate-like structures (see Fig. 3A-F). There are usually two smaller "plates" that are arranged horizontally, just dorsal to the intercoxal plate (Fig. 3B-F). These paired "plates" (Fig. 3G, a) are actually parts of a more or less bipartite, single lobe located within the ventral gap between successive somites. This lobe seems to arise from the posterior side of the gap, namely, 
the anterior border of a given somite; hence, I call it the "anterior lobe." Most of the gap appears to be made of articulation membrane. The anterior lobe does not appear, at least externally, to be well sclerotized, although it may have proximal, internal sclerites that appear as two "plates" in light microscopy. Anterior lobes are not always seen on an undissected thorax because the gaps in which they are located are usually very narrow or even closed (Fig. 5B). In one specimen examined with SEM, the bipartite anterior lobe of the first thoracic somite is seen to be prominently exposed (Fig. 7D); this is an exceptional case and most anterior lobes are smaller than this.

Apart from the anterior lobe, there are at most three more or less plate-like structures between the dissected legs. The "intercoxal plate" is the anteriormost one (Fig. 3G, b). It is a sclerotized plate that is well defined basally and occasionally bears a shallow notch at the middle of the free edge (Figs 3G, 5B, 7D). Its lateral sides are in contact with the inner edges of the coxae of the paired legs proximal to the coxal bosses.

When observed with a light microscope, the intercoxal plate often appears as if it were fringed with a narrow membrane along its free edge (Fig. 3G-E). With carefully focusing, this membrane is resolved as a plate-like structure posterior to the intercoxal plate (Fig. 3G, c). In SEM examination, it is visible as an anterior, transverse fold of the sternite, tightly pressed against the rear of the intercoxal plate (Fig. 7D), although on the first thoracic somite it is not prominent (Fig. 5B). It seems to be less sclerotized than the intercoxal plate and I call it the "intercoxal ridge." Its free edge is either flat or bipartite due to a median, shallow depression. The lateral margins of the intercoxal ridge are connected to the inner edges of coxae proximal to the coxal bosses. Posterior to the intercoxal ridge, there may be a few folds on a flexible area. If present, they are very low and unsclerotized, so there is no chance that any of them could be mistaken for a plate-like structure in a microscopic examination of dissected legs.

Separated from the intercoxal ridge is a prominent, almost rectangular "plate" with somewhat rounded, ventrolateral corners (Fig. 3G, d). Its overall appearance is obvious when viewed from the rear (Fig. 3A), and it does not arise between the paired legs. Actually, it is a ventrally extended ridge formed at the posterior extremity of the sternite of a given somite (Fig. 5B), and it is not directly connected with that somite's legs, even though it might touch their rear side depending on the posture of the animal or movement of the legs (Fig. 7B, D). I call this plate the "posterior ridge" of the sternite. This posterior ridge is occasionally seen, in a microscopic view of dissected legs, to be basally accompanied by a narrow, cuticular "chord" with long bilateral extensions (Fig. 3G). This structure is merely a horizontal view of part of the sternite posterior to the intercoxal ridge. In the middle of the posterior ridge of the sternite is a round area (Fig. 3A, G) which is not a hole but a less sclerotized, translucent area. The lateral portions of the posterior ridge may be somewhat rolled posteriorly (Fig. 7B).

The posterior ridge of the sternite of a given somite is widely separated from 
the intercoxal ridge of the same somite if the animal's trunk is not bent (Fig. 7D). However, when the trunk is bent, the posterior ridge of the sternite is tightly pressed against the intercoxal ridge of its somite (Fig. 5B). The flexible area of the sternite that connects the intercoxal ridge to the posterior ridge enables this. The lateral portions of the posterior ridge continue anterodorsally, and their outer ends partly bound a deep, transverse depression on each side (Fig. 7D). The lateral extremity of each depression sits near the midwidth of the coxa of the leg on that side. Such depressions are not visible on a bent trunk, at least not externally; they would become collapsed or remain as closed pockets in such a case, and probably during backward strokes of the legs as well.

Discussion. Among the named species of Hansenocaris (Itô, 1985, 1986; see also Itô \& Ohtsuka, 1984, and Itô, 1984), the present new species resembles $H$. pacifica Itô, 1985, in many characteristics, e.g., the general shape of the carapace without rostral projection, the antennule with strong claw, the bifurcate paraocular processes of moderate size, the four-segmented abdomen with short furcal rami, and the presence of rectangular plates on the last abdominal somite. However, the new species differs from the latter in some characters. The new species has no mesh-like texture on its cephalon or abdomen, while those parts of the body in the latter species have a prominent mesh-like texture (Itô \& Ohtsuka, 1984). The dorsal plates of the last abdominal somite in the new species are distinctly shorter than those of $H$. pacifica, and each row of dorsal plates consists of as many as ten plates, while only six plates occur in each row in $H$. pacifica.

The present new species differs from all of the previously named species of $\mathrm{Han}$ senocaris in having three-segmented endopods on legs 2-6. With respect to the endopod segmentation of these legs, the new species agrees with a specimen recorded from Denmark by Bresciani (1965), two of the four specimens recorded from the Bahamas by Schram (1970), and two specimens recorded from Denmark and Greenland by Grygier (1987c). The previously named species and the other two specimens recorded from the Bahamas have two-segmented endopods on their legs 2-6. This difference in the endopod segmentation is probably not due to individual variation as suggested by Grygier (1987b, p. 270). The endopod segmentation does not appear to be variable within a single species, as far as is seen in the species known from Japan. Furthermore, the two types of endopod segmentation recognized in Schram's material from the Bahamas can be understood to reflect specific differences. Two specimens labeled as No. 2 and No. 3 by him are almost identical in the armature of their furcal rami, and they both have two-segmented endopods. The specimen labeled as No. 1 is different from the former two specimens in the armature of the furcal rami, and its endopods are three-segmented. The specimen labeled as No. 4 is known to have three-segmented endopods but its furcal armature is unknown. These observations suggest that Schram's material contained at least two species.

The present study of $H$. furcifera is the first to demonstrate the presence of a 
possible penis in facetotectans. Grygier (1987c) described a pair of small spines on the first abdominal somite of his cypris y specimen from Greenland. He suggested that these structures could be rudimentary genitalia. In contrast, the examined specimens of $H$. furcifera show a much more prominent structure on the first abdominal somites, and something similar to the one observed by Grygier on the second.

The supposed penis of this new species (Fig. 6) resembles penes of certain ascothoracidans, e.g., Cardomanica longispinata (Grygier) (Grygier, 1984), and Waginella sandersi (Newman) (see Grygier, 1987a), though that of the new species is much simpler. In these ascothoracidans the penis is a single, median process arising from the first abdominal somite, consisting of a main shaft that ends anteriorly in a pointed apex, where two rami arise. These characteristics are also recognized in the penis of $H$. furcifera. This similarity in penes fills at least a part of the morphological gap between the Facetotecta and Ascothoracida (cf. Bresciani, 1965; Schram, 1970; Itô, 1987a; Grygier, 1987c) and it reinforces my reasons for not creating separate genera other than Hansenocaris within the Facetotecta (Itô, 1986, p. 339).

I have not yet found any specimen without a penis that is otherwise identifiable as $H$. furcifera. Since it is known that certain ascothoracidans have penis even in functional females, the presence of the penis in the examined specimens of $H$. furcifera does not necessarily mean that they are male. Identification of the sexes in facetotectan cyprids, including the possibilities that they are hermaphroditic or sexually indeterminate, is still pending.

The penis found in $H$. furcifera is not articulated to the abdominal somite; rather, it appears to be an extension of the sternite (Fig. 5D). In addition, the sternite of the second abdominal somite has also a posterior extension with a bifid apex, somewhat similar to the apex of the penis. These observations suggests that the penis of the Facetotecta is a modified part of the sternite, not a structure directly derived from paired limbs (cf. Grygier, 1987c).

The antennule of the present new species resembles those of $H$. pacifica and of other facetotectan cyprids from Denmark (Bresciani, 1965; Grygier, 1987c), the Bahamas (Schram, 1970), and Greenland (Grygier, 1987c) in having a strong, arched claw. Among these, Bresciani's material is unique because its antennule has as many as four apical segments distal to the claw-bearing segment, while only two segments occur there in the other cyprids.

Apart from the actual number of segments, there necessarily exist some segments distal to the claw-bearing segment of the antennule. This apical part of facetotectan antennules is called the "palp" by Grygier (1987b). He suggests that the palp can be equated to so-called "proximal sensory process" known in some ascothoracidans (Grygier, 1987b, p. 101). I agree with his suggestion in principle. However, I hesitate to accept his suggestion as it is, because this ascothoracidan "process" is occasionally said to consist of two elements attached to more or less separate places of the claw-bearing segment (e.g., Grygier, 1987b, Fig. 6). In such cases, only the distal "element" should be equated to the palp in facetotectan antennules. The other, proximal "element" may not be equated to the palp. It may 
be an aesthetasc of a separate origin, because Tessmann's larvae (a kind of ascothoracid larvae) recently reported from Hawaii are known to have prominent, proximal aesthetascs in addition to a single, usual "proximal sensory process" that is located distally (Grygier, 1988).

The present new species has a deep recess on the claw-bearing segment of the antennule. Presence of a similar structure is known also in the facetotectan cyprids reported by Grygier (1987c). There is no doubt that the recess functions as scabbard for the claw of this segment (Fig. 4G). This suggests that the particular part forming the recess in facetotectan antennules is, at least in part, homologous to the claw guard in various ascothoracidans. The claw guards are usually trough-shaped because of the presence of lateral flange, and are very pronounced structures arising from antennular, claw-bearing segment (see Grygier, 1987b). Based upon the present observation of facetotectan antennules, I consider that the claw guard is a markedly extended, special part of the claw-bearing segment, not a separate segment attached to the claw-bearing segment.

The cyprids of this new species have a mouth opening at the posterior base of the labrum, like that described for unnamed specimens from Greenland and Denmark (Grygier, 1987c; Bresciani, 1965, unidentified as such). However, this does not necessarily mean that all facetotectan species have a mouth at their cypris $y$ stage. The present new species is known to have a mouth during naupliar stages and is not completely lecithotrophic. In fact, this is a very rare case among the facetotectan larvae that I have observed so far (Itô, 1987a).

In the facetotectan cyprids, the proximal portions of the legs and associated structures were described in detail by Grygier (1987c). With respect to these structures, I have found some discrepancies between the results of my observations and those reported by him. I will enumerate them for the sake of further comparative morphological studies, although it is, for the time being, unlikely that these structures will play an important role for taxonomy within the Facetotecta.

Grygier recognizes "three or four outer sclerites" on the proximal portion of the coxa of each leg. What he calls sclerites "a", "b" and "c" correspond to the sclerotized parts of the first, second, and third coxicules in my sense, respectively. He recognizes the presence of sclerite " $\mathrm{d}$ ", which occurs posteriorly at the same level as sclerite "c" and is separated from it (except in leg 6) by thin cuticle. In my specimens examined with SEM, I found no such sclerite and no interruption of the sclerotized part of the third coxicule, just a shallow pit, which might correspond to the "hole" observed in sclerite "c" by Grygier. On the posterior side of the coxa, the three coxicules meet at a particular spot that I have designated as the pivot hole. Neither such a hole nor any structure equivalent to it was reported for the Greenlandic specimen, whose leg bases were only studied from the side.

There is little doubt that sclerite "e" in the sense of Grygier corresponds to the pleural slat recognized in the present material. In the new species, the first four thoracic somites have pleural slats, but an equivalent structure is only known to occur on the fourth thoracic somite in the Greenlandic specimen, because the lateral 
body wall of the first three somites was hidden by the carapace. Sclerites similar to the pleural slat are known to occur in an ascothoracidan genus, Thalassomembracis Grygier, although they have been reported to sit on the thoracomeres 2-5 (Grygier, 1984).

A comparison of the plate-like structures located between paired legs presents a morphological problem. Grygier (1987c) reports the presence of two sclerites between each pair of legs, which he calls anterior and posterior transverse sclerites. These anterior and posterior sclerites are seemingly identifiable with the intercoxal plate and the intercoxal ridge, respectively. Nevertheless, they are greatly different morphologically.

Grygier's posterior transverse sclerite is flat, "with its rear end rolled into a tube that connects the legs and braces the coxal bosses." None of the features mentioned in the quoted passage is recognized in the intercoxal plate. It is likely that an intercoxal plate appears as a sclerotized cuticular tube in sagittal sections of the animal, because it is well-sclerotized at least externally. Hence, the rolled rear end ("tube") of the posterior sclerite in the sense of Grygier might be equivalent to the intercoxal plate in my sense. If so, the identity of the anterior transverse sclerite should become another problem. He mentions that "thin cuticle connects the tube to the next segment's anterior transverse sclerite." This observation suggests a possibility that the so-called anterior transverse sclerite is equivalent to the anterior lobe in my sense, though morphological differences are present between them. For example, the anterior sclerite is reported to have a pair of depressions and articulate with the rear basal corners of the preceding limb pair's 'a' sclerites, but no such characteristics are recognized in the anterior lobe in the new species. If they were actually equivalents despite of these morphological differences, in front of the anterior transverse sclerite on a given somite there should be the posterior ridge of the sternite of the preceding somite. However, no structure equivalent to the latter is recognized in Grygier's material. It is now an open question whether these discrepancies reflect actual morphological differences or not.

Diagnosis. Cypris y. Carapace with evenly rounded front, with no rostral projection, with almost longitudinal ridges, no mesh-like texture. Branches of bifurcate paraocular process not huge. Abdomen consisting of four somites, the first of which bears medially a possible penis; the last having rows of rectangular plates, the dorsal rows of which each consists of about 10 plates. Furcal rami short, two-annulated. Antennule armed with movable, strong claw on the second segment. Each endopod of posterior five pairs of legs three-segmented.

Etymology. The specific name "furcifera" depicts that this animal bears a forkshaped, possible penis.

\section{Acknowledgments}

I express my gratitude to Dr. M.J. Grygier for reading the manuscript. This work is supported 
in part by a Grant-in-Aid for Scientific Research, No. 62540567, from the Ministry of Education, Science and Culture, Japan.

\section{References}

Bresciani, J. 1965. Nauplius " $y$ " Hansen. Its distribution and relationship with a new cypris larva. Vidensk. Medd. fra Dansk Naturh. Foren., 128: 245-258, pl. XL.

Grygier, M.J. 1984. Ascothoracida (Crustacea: Maxillopoda) parasitic on Chrysogorgia (Gorgonacea) in the Pacific and western Atlantic. Bull. Mar. Sci., 34: 141-169.

- 1987a. Reappraisal of sex determination in the Ascothoracida. Crustaceana, 52: 149162.

1987b. Nauplii, antennular ontogeny, and the position of the Ascothoracida within the Maxillopoda. J. Crust. Biol., 7: 87-104.

- 1987c. New records, external and internal anatomy, and systematic position of Hansen's y-larvae (Crustacea: Maxillopoda: Facetotecta). Sarsia, 72: 261-278.

- 1988. Larval and juvenile Ascothoracida (Crustacea) from the plankton. Publ. Seto Mar. Biol. Lab., 33: 163-172.

Itô, T. 1984. Another cypris y from the North Pacific (Crustacea: Maxillopoda). Publ. Seto Mar. Biol. Lab., 29: 365-372.

1985. Contributions to the knowledge of cypris y (Crustacea: Maxillopoda) from the North Pacific. Spec. Publ. Mukaishima Mar. Biol. Stat., 1985: 113-122.

- 1986. A new species of "cypris y" (Crustacea: Maxillopoda) from the North Pacific. Publ. Seto Mar. Biol. Lab., 31 : 333-339.

- 1987a. Y-larvae-enigmatic crustacean larvae. Ann. Rep. Seto Mar. Biol. Lab., 1: 5258. (In Japanese)

- 1987b. Proposal of new terminology for the morphology of nauplius y (Crustacea: Maxillopoda: Facetotecta), with provisional designation of four naupliar types from Japan. Zool. Sci., 4: 913-918.

\& S. Ohtsuka. 1984. Cypris y from the North Pacific (Crustacea: Maxillopoda). Publ. Seto Mar. Biol. Lab., 29: 179-186.

—_ \& M. Takenaka. 1988. Identification of bifurcate paraocular process and postocular filamentary tuft of facetotectan cyprids (Crustacea: Maxillopoda). Publ. Seto Mar. Biol. Lab., 33: 19-38.

Schram, T.A. 1970. Marine biological investigations in the Bahamas. 14. Cypris y, a later developmental stage of nauplius y Hansen. Sarsia, 44: 9-24. 